\title{
Cold Storage of Atractylodes ovata Shoot Cultures and Evaluation of the Regenerated Plants
}

\author{
Noboru HIRAOKA ${ }^{1 *}$, Jung-In CHANG ${ }^{1 a}$ and Indra Dutt BHATT ${ }^{1}$ \\ 'Department of Plant Resources and Biotechnology, Faculty of Applied Life Sciences, Niigata University of \\ Pharmacy and Applied Life Sciences, 265-1, Higashijima, Niitsu-shi 956-8603, Japan \\ Present address: ${ }^{a}$ Unigen Co., Daeyang Koreana Bldg, 182-2 Bang Yi-dong, Songpa-Gu, Seoul, Korea \\ *Corresponding author E-mail address: hiraoka@niigata-pharm.ac.jp
}

Received 18 April 2003; accepted 1 July 2003

\begin{abstract}
Atractylodes ovata (Compositae) shoot cultures were put into cold storage and the regenerated plants then evaluated. All the in vitro shoot cultures of $A$. ovata DC. survived cold storage at $2{ }^{\circ} \mathrm{C}$ for up to 24 months. The cold-stored shoots were then multiplicated and rooted under normal culture conditions. The regenerated plants were cultivated in the field for one or two years and various biological and biochemical characteristics were compared with a control plant. Statistical analysis of the data showed no significant differences between the two groups in most morphological traits and the contents of the atractylon, a sesquiterpene component of essential oil in the rhizome. These results demonstrate that non - frozen cold storage of in vitro shoot cultures can be used as a germplasm preservation system for short - or medium-duration without deterioration of their biological and biochemical characteristics.
\end{abstract}

Key words: atractylon, Compositae, essential oil, germplasm preservation, micropropagation, refrigeration.

\begin{abstract}
Abbreviations
BA, 6-benzyladenine; IAA, indole-3-acetic acid; LS, Linsmaier and Skoog (1965) medium; NAA, 1-naphthaleneacetic acid.
\end{abstract}

Atractylodes ovata DC. (A. macrocephala Koidzumi) is a valuable perennial medicinal plant distributed in China. Its rhizome is listed in Japanese and Chinese Pharmacopoeia and used as a digestive, diuretic, and antihidrotic (Tang and Eisenbrand, 1992). Atractylon is a major sesquiterpenoid component of essential oil in the rhizome of $A$. ovata and has various pharmacological activities, including antihepatotoxicity (Kiso et al., 1983; Yamahara et al., 1983), antiulcer action (Matsuda et al., 1991) and cytotoxic activity (Wang et al.,2002). A. ovata is generally propagated by seeds (Fukuda et al., 1992) and requires cross fertilization for seed production. Consequently, a wide range of variation has been observed in the morphological and chemical characteristics of this species (Fukuda et al., 1997). Furthermore, A. ovata is easily hybridized with other Atractylodes species (Fukuda et al., 1995). Therefore, micropropagation seems an alternative option for producing true to type plants and also for preserving the traits of a mother plant. Sung and Zheng (1989) and Hatano et al. (1990) reported the clonal propagation of $A$. ovata. However, no attempt has been made for in vitro preservation and assessment of regenerated plants. In our previous study using $A$. lancea, we reported plant regeneration starting from floral buds or shoot tips (Hiraoka et al., 1984; Hiraoka and Tomita, 1990), cold storage of shoot cultures (Hiraoka, 1992) and biological and chemical evaluation of plants regenerated from refrigerated shoots (Hiraoka and Kagoshima, 1993). The present communication describes the non-frozen method for medium-term preservation of in vitro A. ovata shoots and assessment of regenerated plants. The primary focus was the effect of long-term cold storage of shoots on accumulation of secondary metabolites or pharmacologically active principles in plants raised from cold - stored shoot cultures.

A. ovata plants were a kind gift from Dr. T. Kimura, Daiich College of Pharmaceutical Sciences and were maintained at the Medicinal Plant Garden, Niigata University of Pharmacy and Applied Life Sciences in Niigata city, Japan (Plant registration No. 913).

Throughout the present study, Linsmaier and 
Skoog (1965) medium containing 3\% sucrose and $0.8 \%$ agar was used as the basal medium. A culture tube (o.d. $13 \times 130 \mathrm{~mm}$ ) containing $20 \mathrm{ml}$ of agar medium was capped with a silicone sponge and autoclaved for $20 \mathrm{~min}$ at $121^{\circ} \mathrm{C}$. All the cultures were incubated at $25^{\circ} \mathrm{C}$ under fluorescent lamps producing an irradiance of $37 \mu \mathrm{mol} \mathrm{m} \mathrm{m}^{-2} \mathrm{~s}^{-1}$ at culture level over a $16 \mathrm{~h}$ photoperiod, except where otherwise stated. The meristematic parts having two leaf primordia of about $0.5 \mathrm{~mm}$ diameter were removed aseptically from two shoot tips of a single plant of $A$. ovata and inoculated on the basal medium fortified with $10 \mu \mathrm{M}$ NAA and $1 \mu \mathrm{M}$ BA. Each $5 \mathrm{~mm}$ long shoot emerged was transferred to the shoot proliferating medium consisting of basal medium containing $1 \mu \mathrm{M}$ IAA and $10 \mu \mathrm{M}$ BA. Shoot cultures were maintained by subculturing every 1-2 months on the shoot proliferation medium under normal culture conditions. Shoot innocula were trimmed to about $1 \mathrm{~cm}$ in length (weight about $0.06 \mathrm{~g}$ ) and inoculated singly. Ten shoots were cultured on shoot proliferation medium for 11 days before they were cold-stored at $2{ }^{\circ} \mathrm{C}$ under continuous illumination from a fluorescent lamp at

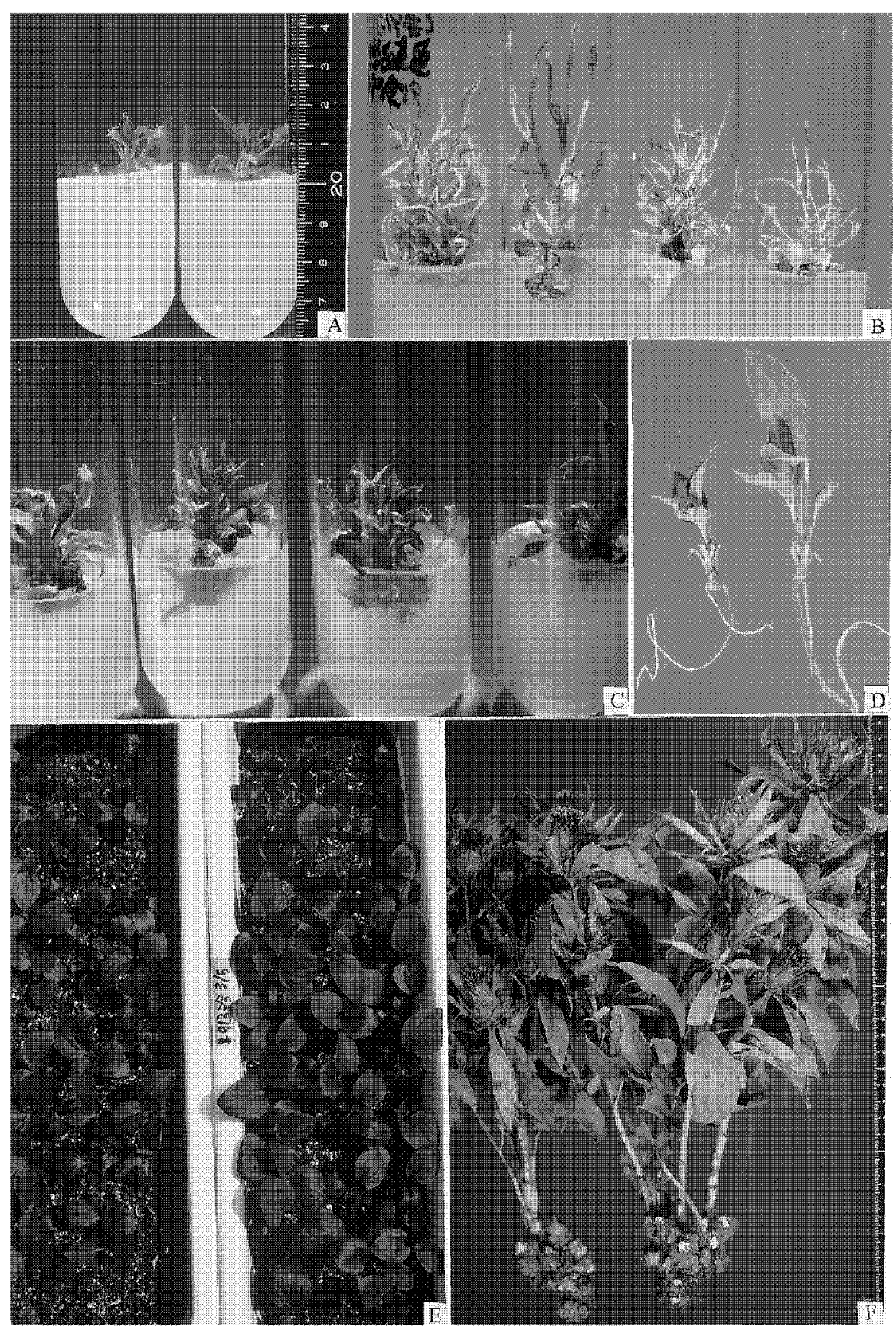

Fig. 1 Cold storage of in vitro shoots and field performance of regenerated plants of Atractylodes ovata. (A) Eleven day old precultured shoots for cold storage. (B) 24-month - old cold stored shoots. (C) Recultured shoots. (D) Rooted shoots. (E) Regenerated plants in planters and $(\mathrm{F}) 2$-year-old regenerated plants cultivated in the field. $(\mathrm{C}-\mathrm{F})$ Left to right: control and cold stored. 
irradiance of $5 \mu \mathrm{mol} \mathrm{m} \mathrm{m}^{-2} \mathrm{~s}^{-1}$. After one and two years of storage, each of 5 cultures was withdrawn from the cold room. During this period, control cultures were maintained by regular subculture under normal conditions. The cold-stored shoots were recultured for shoot proliferation. After three passages of subculturing on the shoot proliferation medium, cold-stored shoot cultures were inoculated on the rooting medium (LS agar medium containing no plant growth regulators) and incubated for 40 days. Shoots without cold storage inoculated into same rooting medium served as the control.

The rooted shoots raised from the shoots of both groups were transplanted into planters filled with a mixture of soil-vermiculite-sand (1:1:1 by volume) and covered with vinyl sheets for the first 10 days to maintain high humidity. After one month of cultivation in planters, the plantlets were transplanted to an experimental field in April 2001 for the first year field trial. The crops were harvested in early November of the same year and various traits were recorded. Rhizomes were dried at $60^{\circ} \mathrm{C}$ and stored at room temperature in a vinyl bag with "AGELESS” (Mitsubishi Gas Chemical Co. Inc.), an oxygen consuming reagent, till chemically analyzed. On the last day of November of the same year, all the remaining rhizomes were dug out of the soil and the roots discarded. The rhizomes were arranged stem - side up in a wooden box, which was buried underground outdoors until the following spring. In early March 2002, the rhizomes were taken out of the wooden box and planted again for the second year field trial. All the plants were harvested on the last day of October of the same year and treated as above (Ministry of Health and Welfare, Japan, 1996). The atractylon content in the dry rhizome was determined by the gas chromatographic method, as reported previously (Hiraoka, 1995). The statistical significance of differences between the control and cold-treated groups were assessed using Student's t test.

The shoot culture of $A$. ovata was successfully established from shoot tips and subcultured into shoot proliferation medium (LS $+10 \mu \mathrm{M} \mathrm{BA}+1$ $\mu \mathrm{M}$ IAA). The average shoot proliferation rate in a one-month culture period was 3.2-4.0 shoots/explant during the first five years, which decreased to 2.0-3.0 shoots/explant thereafter. Shoots maintained for one year served as the plant material for the cold preservation experiments. Shoots were precultured for 11 days on the shoot proliferation medium before the start of cold storage at $2^{\circ} \mathrm{C}$ (Fig. 1A). The control shoot culture was maintained by subculturing under normal culture conditions without refrigeration.

Cold-stored shoots for 1 year were $5.0 \mathrm{~cm}$ long,

Table 1 Field trials of Atractylodes ovata plants regenerated from cold-stored shoot cultures.

\begin{tabular}{|c|c|c|c|c|}
\hline \multirow{2}{*}{ Characteristics } & \multicolumn{2}{|c|}{ First year of cultivation $(n=12)$} & \multicolumn{2}{|c|}{ Second year of cultivation $(n=10)$} \\
\hline & Control & Cold-stored & Control & Cold-stored \\
\hline \multicolumn{5}{|l|}{ Aerial parts } \\
\hline Plant height $(\mathrm{cm})$ & $22 \pm 5$ & $20 \pm 3$ & $31 \pm 3$ & $29 \pm 6$ \\
\hline Fresh weight (g) & & & $59 \pm 31$ & $57 \pm 44$ \\
\hline Diameter of a stem (mm) & $6.3 \pm 1.3$ & $6.4 \pm 1.5$ & $7.5 \pm 2.1$ & $8.1 \pm 2.2$ \\
\hline Number of nodes & & & $17.5 \pm 2.5$ & $19.6 \pm 2.3$ \\
\hline Number of branches & $3.1 \pm 2.4$ & $1.7 \pm 1.8$ & $8.2 \pm 3.9$ & $5.9 \pm 3.2$ \\
\hline Leaf length (mm) & $82 \pm 17$ & $78 \pm 8$ & $95 \pm 11$ & $96 \pm 8$ \\
\hline Leaf width (mm) & $28 \pm 5$ & $26 \pm 4$ & $55 \pm 12$ & $48 \pm 14$ \\
\hline Capitulum length (mm) & $35 \pm 2$ & $33 \pm 3$ & $41 \pm 4$ & $39 \pm 3$ \\
\hline Capitulum width (mm) & $22 \pm 1$ & $20 \pm 2$ & $18 \pm 2$ & $19 \pm 3$ \\
\hline Floret length (mm) & $34 \pm 1$ & $33 \pm 1$ & $30 \pm 2$ & $29 \pm 2$ \\
\hline \multicolumn{5}{|l|}{ Rhizome } \\
\hline Fresh weight $(\mathrm{g})$ & $10.6 \pm 3.1$ & $15 \pm 5.2^{*}$ & $42.2 \pm 14.3$ & $42.7 \pm 15.2$ \\
\hline Dry weight (g) & $4.3 \pm 1.4$ & $6.2 \pm 2.3^{*}$ & $12.2 \pm 5.0$ & $13.2 \pm 5.8$ \\
\hline Dry wt./Fresh wt. ratio & $40.3 \pm 1.4$ & $40.9 \pm 2.1$ & $29.3 \pm 8.2$ & $31.1 \pm 6.9$ \\
\hline Atractylon content (\% DW) & $0.7 \pm 0.21$ & $0.62 \pm 0.14$ & $1.83 \pm 0.32$ & $1.73 \pm 0.89$ \\
\hline
\end{tabular}

The plants were raised from shoot cultures stored at $2{ }^{\circ} \mathrm{C}$ for 24 months. Data represent mean $\pm \mathrm{SD}$. Fresh weight and the number of nodes were not recorded in the first year.

* Significantly different from control at $p<0.05$. 
weighed $0.87 \mathrm{~g}$, and consisted of 7.8 shoots over an average of 5 cultures. They appeared healthy and proliferated as vigorously as the control group when recultured. At the third passage of reculture following the cold storage, no significant difference in proliferation rate was found between the coldstored strain ( $3.9 \pm 2.4$ shoots/culture) and the control ( $3.2 \pm 1.5$ shoots/culture).

After two years of cold storage, the remaining five cultures were withdrawn from the cold room (Fig. 1B). All the cultures except one, which was contaminated, survived the cold storage, although the leaves were partly deteriorated. The average weight, height, and number of shoots per culture were $1.17 \pm 0.65 \mathrm{~g}, 3.8 \pm 1.9 \mathrm{~cm}$, and $4.3 \pm 4.0$, respectively. No significant difference was found in the proliferation rate between the cold-stored strain $(5.4 \pm 1.9)$ and the control $(4.4 \pm 2.3)$ at the second passage of reculture (Fig. 1C). The rooting ratio of the shoots on the rooting medium was $98 \%(97 / 99)$, whereas that of the control was $87 \%(84 / 97)$. The number of roots per shoot was significantly $(p<0.05)$ higher in cold-stored group $(4.0 \pm 1.6)$ than the control $(2.8 \pm 1.8)$, however, no significant difference was found in root length (control, $40 \pm 17$ $\mathrm{mm}$ and cold-stored strain, $48 \pm 11 \mathrm{~mm}$ ) (Fig. 1D). The percentage of control plants that survived in the planters was $24 \%$ while $42 \%$ of the cold stored strain survived (Fig. 1E). The data from the first and second year of cultivation are shown in Table 1. The characteristics of both groups were very similar, except for rhizome weight (Fig. 1F). The second year crops were larger and heavier than the first year ones in vegetative organs. In particular, rhizome weight and atractylon content were markedly higher. No deviation was observed in the morphology of the leaf, bract and floret or rhizome of the two groups. The capitulum was bigger than the other species of Atractylodes and had fish-bone shaped bracts and reddish purple florets as in the original plants.

The reproducibility of any protocol depends on successful field performance and assessment of micropropagated plants. In previous studies on $A$. lancea, we found that a duration of 24 months seemed to be the limit of cold storage of in vitro shoot cultures under the same conditions as in the present study, since leaves began to deteriorate around this time (Hiraoka and Kagoshima, 1993). In the present study using $A$. ovata, the control and cold stored group showed the same morphological features including compound leaf shape at the middle and lower parts of the stem, characteristic bone shaped bracts, and red-purple florets. Although a difference in rhizome weight was observed between the two groups in the first year crop, this was not the case in the second year crop. Flowering time was also the same between the two groups. The gas chromatogram of $n$-hexane extract of the rhizome was almost the same for the two groups and showed one main peak of atractylon and several small unidentified peaks. No significant differences in content were observed, as previously reported (Hatano et al., 1990; Mizukami et al., 1996; Fukuda et al., 1997; Hiraoka et al., 1997).

The present findings coincide with the data obtained with $A$. lancea (Hiraoka and Kagoshima, 1993) and clearly demonstrate that non-frozen cold storage of in vitro $A$. ovata shoots does not affect the morphological and biochemical features of the regenerated plants. Consequently, slow growth storage of shoot cultures at a temperature of a little above $0{ }^{\circ} \mathrm{C}$ provides a dependable method for the medium-term preservation of plant resources without adverse effects on various characteristics, including secondary metabolite accumulation.

\section{Acknowledgments}

The authors thank Professor T. Kimura for the plant material and Ms. C. Shinohara and Ms. H. Ogata for their technical assistance. This work was supported in part by a Grant-in-aid for Scientific Research (c) 11672232 from the Ministry of Education, Science, Culture, and Sports (Japan) to NH and by a Grant-in-aid for Scientific Research P-02820 from the Japan Society for the Promotion of Science to $\mathrm{NH}$ and IDB.

\section{References}

Fukuda, T., Aragane, M., Yoshizawa, M., Suzuki, Y., Tanaka, H., 1992. Studies of cultivation of Atractylodes ovata. I. Some aspects of seed production. Jpn. J. Pharmacog, 46: 240- 245 (in Japanese).

Fukuda, T., Nakajima, J., Aragane, M., Yoshizawa, M., Suzuki, Y., Shimizu, T., 1995 Studies of cultivation of Atractylodes ovata. III. Hybridization and the change of morphological characteristics caused by the hybridization. Natural Medicines, 49: 431 - 437 (in Japanese).

Fukuda, T., Nakajima, J., Yasuda, I., Aragane, M., Yoshizawa, M., Suzuki, Y., Shimizu, T., 1997. Studies of cultivation of Atractylode ovata. IV. Individual variation of external morphology and individual difference of amount of growth and contents of chemical substances. Natural Medicines, 51: 420-426 (in Japanese).

Hatano, K., Shoyama, Y., Nishioka, I., 1990. Clonal propagation of Atractylodes japonica and $A$. ovata by tip tissue culture and the atractylon content of clonally propagated plants. Planta Med, 56: $131-132$.

Hiraoka, N., 1992. Cold storage of Atractylodes lancea shoot cultures. Jpn. J. Pharmacog., 46: 206-209.

Hiraoka, N., 1995. Intra-plant distribution of essential oil 
components and oil accumulation tissues in Atractylodes lancea. Natural Medicines, 49: 168-171.

Hiraoka, N., Kagoshima, K., 1993. Morphological and chemical evaluation of Atractylodes lancea plants raised from refrigerated shoot cultures. Plant Tissue Cult. Lett., 10: $169-171$.

Hiraoka, N., Matoba, K., Ogawa, N., Kagoshima, K., 1997. Atractylon is a major sesquiterpene in young Atractylodes lancea plants raised from shoot cultures. Natural Medicines, 51: 417-419.

Hiraoka, N., Tomita, Y., 1990. Botanical and chemical evaluation of Atractylodes lancea plants propagated in vitro and by division of the rhizome. Plant Cell Rep., 9: $332-334$.

Hiraoka, N., Yamada, Y., Kodama, T., Tomita, Y., 1984. In vitro propagation of Atractylodes lancea. Plant Cell Rep., 3: 85-87.

Kiso, Y., Tohkin, T., Hikino, H., 1983. Antihepatotoxic principles of Atractylodes rhizomes. J. Nat. Prod., 46: $651-654$.

Linsmaier, E. M., Skoog, F., 1965. Organic growth factor requirements of tobacco tissue cultures. Physiol. Plant., 18: $100-127$.

Matsuda, H., Li, Y., Taniguchi, K., Yamahara, J,, Tamai, Y., 1991. Imaging analysis of antiulcer action and the active constituent of Atractylodis Rhizoma. Yakugaku Zasshi, 111: 36-39 (in Japanese).

Ministry of Health and Welfare, Japan, 1996. Medicinal plants - Cultivation and Evaluation, 13-24. Yakuji Nippo-sha, Tokyo.

Mizukami, H., Shimizu, R., Kohda, H., Kohjyouma, M., Kawanishi, F., Hiraoka, N., 1996. Restriction fragment length polymorphisms of rDNA and variation of essential oil composition in Atractylodes plants. Biol. Pharm. Bull., 19: $577-580$.

Sung, P. L., Zheng, J. G., 1989. Tissue culture of large- head Atratcylodes (Atractylodes macrocephala) and ternate pinellia (Pinellia ternata). Chinese Traditional and Herbal Drugs, 20: $315-317$ (in Chinese).

Tang, W., Eisenbrand, G., 1992. Chinese Drugs of Plant Origin. Springer - Verlag, Berlin.

Wang, C. C., Chen, L. G., Yang, L. L., 2002. Cytotoxic activity of sesquiterpenoids from Atractylodes ovata on leukemia cell lines. Planta Med., 68: 204-208.

Yamahara, J., Matsuda, H., Kobayashi, M., Sawada, T., Fujimura, H., 1983. Biologically active principles of crude drugs. Pharmacological evaluation of "Baizhu" and "Chongzhu" (II). Jpn. J. Pharmacog, 37: 17-20 (in Japanese). 